Vlatka Rubinjoni Strugar ${ }^{1}$

UDK: 371.3::811.134.2'367.625

Universidad de Alcalá

811.163.41: 811.134.2

Alcalá de Henares (España)

https://doi.org/10.18485/imp.2017.ch.7

Departamento de Filología

Comunicación y Documentación

\title{
LA IMPORTANCIA DE COMBINAR EL ANÁLISIS CONTRASTIVO Y EL ANÁLISIS DE ERRORES EN LOS ESTUDIOS QUE CONTRASTAN EL ESPAÑOL Y EL SERBIO
}

Desde una perspectiva teórica, los resultados del Análisis contrastivo se utilizan para numerosas investigaciones, útiles, sobre todo, para la Tipología lingüística. Desde una perspectiva aplicada, la Lingüística contrastiva constituye una herramienta práctica para la enseñanza de lenguas extranjeras, ámbito en el que, actualmente, se emplea para desarrollar estrategias de aprendizaje, pues ayuda a desarrollar la memoria y mejorar la comprensión; asimismo, cumple importantes objetivos didácticos plasmados en la elaboración de material didáctico, incluidas gramáticas contrastivas. El Análisis de errores, como un nuevo modelo de la Lingüística contrastiva, surgió como consecuencia de la falta de validez predictiva del Análisis contrastivo. En nuestro trabajo justificamos la vitalidad de la Lingüística contrastiva y de sus dos métodos, el Análisis contrastivo y el Análisis de Errores, así como su idoneidad para contrastar el español y el serbio.

Palabras clave: lingüística contrastiva, análisis contrastivo, análisis de errores, estrategias de aprendizaje, español, serbio

\section{Introducción}

La Lingüística contrastiva ha pasado por varias etapas en el transcurso de su desarrollo. Hubo un período en el que alcanzó su auge y disfrutó del pleno entusiasmo de los lingüistas, luego sufrió duras críticas y, al final, ha experimentado su propio resurgimiento, relacionada con una variedad de campos de estudio, tanto teóricos como aplicados. Los estu-

1 vlatka.rubinjoni@edu.uah.es 
dios teóricos se han relacionado con la Lingüística tipológica y universalista y con la Pragmática contrastiva, mientras que los aplicados se extienden prioritariamente a la pedagogía de la lengua. Debido a que el objetivo de este artículo es justificar la vitalidad de la Lingüística contrastiva junto con sus dos modelos, el Análisis contrastivo y el Análisis de errores, hemos optado primeramente por realizar un repaso histórico sobre la Lingüística contrastiva, siguiendo un orden cronológico. En segundo lugar, nos referimos a las críticas del Análisis contrastivo y al surgimiento del Análisis de errores como consecuencia de dichas críticas. En tercer lugar, exponemos aspectos positivos del Análisis contrastivo y, en cuarto lugar, las razones de su revitalización actual. Tras ello, nos referimos a los estudios contrastivos entre el serbio y el español. Finalmente, justificamos la importancia de realizar el Análisis de errores junto con el Análisis contrastivo, exponiendo resultados de nuestra investigación, que atañe a los errores de concordancia en la expresión escrita de los estudiantes serbios de español como lengua extranjera.

\section{Surgimiento y períodos de la lingüística contrastiva}

Las lenguas se han comparado desde tiempo inmemorial debido a perennes contactos entre los pueblos y sus culturas, tanto de forma intuitiva y natural como de manera sistemática. Esta última se ve manifestada en traducciones de numerosos textos latinos eclesiásticos y filosóficos medievales y renacentistas. Según Krzeszowski (1990: 1-2), los estudios contrastivos se remontan a fechas en torno al año 1000, cuando el abad inglés Aelfric escribió su Gramática de latín e inglés basándola en un supuesto implícito de que el conocimiento de la gramática de una lengua puede ayudar al aprendizaje de otra. La profesora Đorđević (2004) va más lejos en la historia y nos habla de la Piedra de Rosetta, la estela egipcia del año 196 a.C., considerada el documento emblemático del antiguo análisis contrastivo por representar un decreto egipcio en tres alfabetos distintos: el texto superior está en jeroglíficos egipcios, la parte intermedia es escritura demótica y el inferior fue inscrito en griego antiguo. 


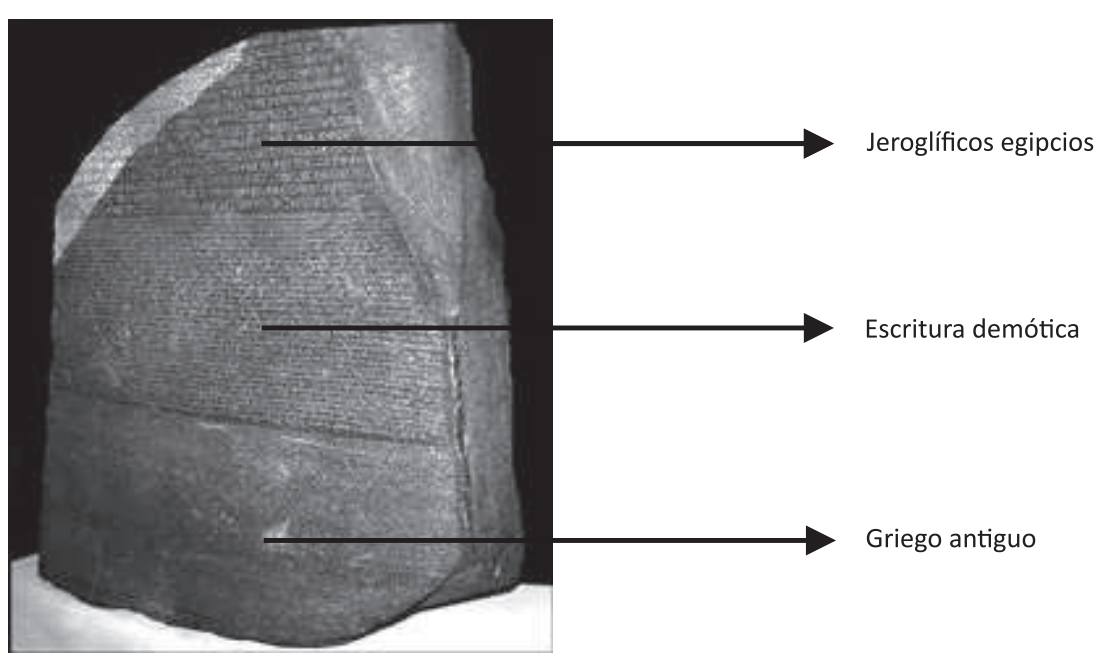

La Piedra de Rosetta, 196 a.C., expuesta en el Museo Británico (Andrews, 1987), es el ejemplar más antiguo de la Lingüística contrastiva. Actualmente, el término Rosetta Stone se utiliza como palabra de referencia para un nuevo campo de conocimiento.

Siguiendo a Martín Morillas (1997), a comienzos del siglo XIX empieza la época del comparatismo histórico-diacrónico, pero los estudios comparativos sincrónicos no aparecen hasta finales de ese siglo y están relacionados con el desarrollo de la fonética. En esto coincide con Đorđević (2004), que vincula los orígenes de la Lingüística contrastiva moderna con el final del siglo XIX, de modo que la trayectoria de los estudios contrastivos, según Đorđević (2004: 12-18), podría dividirse en tres períodos: el período tradicional, el período clásico y el período moderno.

\subsection{Período tradicional}

El período tradicional, que se observa a partir de finales del siglo XIX hasta la Segunda Guerra Mundial, se caracteriza por estudios esporádicos y comparaciones incompletas entre dos o más lenguas. No obstante, surgieron varios trabajos que pusieron de manifiesto los esfuerzos de los lingüistas dirigidos hacia la clasificación de las lenguas del mundo según la tipología lingüística, a la par que se comparaban diacrónicamente. Al mismo tiempo, surgieron obras que han evidenciado estudios más detallados sobre diferencias y similitudes sincrónicas entre las lenguas. Los 
inicios de los estudios contrastivos han sido relacionados con lenguas del territorio europeo: inglés, alemán, holandés, francés y determinadas lenguas eslavas. Posteriormente, surgieron estudios contrastivos en suelo norteamericano, donde se contrastaba el inglés con el chino y, más tarde, el inglés con lenguas indígenas. Cabe añadir que el lingüista norteamericano Leonard Bloomfield destacó en su libro Linguistics (1933: 270) que la tarea de los lingüistas del futuro sería la de comparar categorías de distintas lenguas con el objetivo de descubrir qué categorías tienen valor universal o, al menos, están ampliamente difundidas.

Nos parece significativa esta consideración, que fue postulada hace más de ochenta años, puesto que la cuestión de la universalidad sigue siendo actual en la Lingüística contrastiva.

\subsection{Período clásico}

La época clásica de los estudios contrastivos abarca un período de alrededor de veinte años calculando a partir de la Segunda Guerra Mundial. Este período está marcado por un desarrollo intensivo de investigaciones y una organización más extensa de trabajos. La Lingüística contrastiva se ha convertido, según señala Đorđević (2004), en una disciplina científica, práctica y académica con numerosos resultados. Se acentuaba su aspecto práctico, ya que los resultados del análisis contrastivo han sido significativos tanto en la enseñanza y aprendizaje de lenguas extranjeras como en el estudio del bilingüismo, la traducción o en otras esferas de la Lingüística aplicada. En 1945, la Universidad de Michigan publica la obra de Charles Fries Teaching and Learning English as a Foreign Language en la que, examinando el contraste del inglés con el español, el lingüista norteamericano acentúa la función aplicativa de los estudios contrastivos. Siguiendo a Santos Gargallo (1993: 33), esta obra representa, junto al trabajo de Robert Lado, Linguistics Across Cultures, el fundamento de la investigación del Análisis contrastivo en el marco del enseñanza/aprendizaje de una lengua extranjera. Asimismo, la autora señala que ambos lingüistas proponen la comparación sistemática de dos lenguas: la lengua nativa del estudiante y la lengua que se va a aprender. Siguiendo a Fries (1945: 3-9), el problema principal en el aprendizaje de una nueva lengua

no es aprender el vocabulario. En su opinión la dificultad radica, en primer 
lugar, en el dominio del sistema de sonidos para entender la corriente del discurso, para oír características de sonido distintivas y para aproximar su producción y, en segundo lugar, en el dominio de las características que constituyen la estructura de una lengua. Para conseguirlo, los materiales más eficientes son los que se basan en una descripción científica de la lengua que hay que aprender y, a la par, esta lengua debe ser cuidadosamente comparada y acompañada por una descripción paralela de la lengua materna del estudiante. Además, no es suficiente obtener resultados de un análisis, sino que estos resultados deben estar organizados en un sistema satisfactorio para la enseñanza, suministrándole al alumno material de práctica específico a través del cual pueda dominar el sistema de sonidos, la estructura y el material léxico más útil de la lengua que está aprendiendo. Este dominio se adquiere, siguiendo a Fernández González (1995), a través de la formación de una serie de hábitos que difieren en distinta medida de la lengua materna, siendo parcialmente coincidentes y parcialmente divergentes. En la medida en que existía coincidencia, la adquisición se veía facilitada, transferencia positiva, y en la medida en que existía divergencia, se veía dificultada, transferencia negativa o interferencia. Se acuñó así el término distancia lingüística para dar cuenta del grado de diferencia o similitud entre las lenguas.

No obstante, se considera a Robert Lado uno de los fundadores de la Lingüística contrastiva como disciplina científica y subdisciplina de la Lingüística aplicada. En su libro más influyente, Linguistics across Cultures $(1957)^{2}$, Lado establece la clave del aprendizaje de una lengua, que consiste en contraponer elementos y estructuras de la lengua materna con los de la lengua extranjera, considerando fáciles de aprender los que son similares y difíciles los que son diferentes. Lado, siguiendo investigaciones iniciadas por su profesor ${ }^{3}$ y colega Charles Fries, proporciona los instrumentos necesarios para llevar a cabo dicho proceso y establece el concepto básico del Análisis contrastivo, que consiste en predecir las áreas de dificultad que generarían error durante el proceso de aprendizaje.

2 El título de la versión española es Lingüística Contrastiva. Lenguas y Culturas.

3 Según el Centre for Applied Linguistics de Warwick, Robert Lado fue alumno de Charles Fries en la Universidad de Michigan. 
La meta del Análisis contrastivo ha sido, siguiendo a Santos Gargallo (1993: 35), «construir una gramática contrastiva que establezca una jerarquía de correspondencias de los distintos niveles de gramática, con el fin de graduar las dificultades en el aprendizaje y las posibilidades de interferencia». Siguiendo el mismo concepto, Filipović (1968b: 1-7) afirma en su artículo "Contrastive Analysis in Linguistic Research» que ese tipo de gramática es necesario desde el punto de vista científico, profesional y práctico. Los resultados de estos estudios dedicados a la construcción de una gramática contrastiva pueden ser, según señala Filipović (1968b: 1-7), significativos no solo para el desarrollo de la práctica sino también para el progreso de la teoría lingüística.

Para concluir, Filipović (1968a: 1-5) nos ofrece la definición de Hammer y Rice (1965), expuesta en la introducción de su Bibliografía. La presentamos en original puesto que el objetivo principal del Análisis contrastivo, su función aplicada, ha permanecido hasta el presente a pesar de las críticas sobre su sostenibilidad.

[...] a contrastive structure study is defined as a systematic comparison of selected linguistic features of two or more languages, the intent of which is not to demonstrate or establish genetic of typological relationships, but, typically, to provide teachers and textbook writers with a body of information which can be of service in the preparation of instructional materials, the planning of courses, and the development of classroom techniques.

(Hammer, Rice, 1965: Introduction)

\subsubsection{Lingüística contrastiva vs. Lingüística comparativa}

En el período clásico de la Lingüística contrastiva se planteó la distinción entre los términos Lingüística contrastiva y Lingüística comparativa. Este tema ha sido objeto de numerosas discusiones entre los lingüistas. Siguiendo la aportación de Filipović (1968a: 1-5), aunque el término contrastar no aparece en la mayoría de los trabajos citados por Hammer (1965) en su Bibliografía de la Lingüística contrastiva 4 y prevalece el uso del término comparar, los lingüistas, en efecto, llevaban a cabo 
el contraste sincrónico entre dos o más lenguas, explorando sus estructuras. Filipović (1968a: 1-5) justifica que él mismo, durante el período comprendido entre 1950 y 1963, realizaba análisis sincrónicos y comparaba los elementos fonéticos y sintácticos de la lengua inglesa con los de la croata-serbia, partiendo de la estructura de esta lengua para explicar la del inglés. Su único objetivo era acercar los aspectos fonológicos y sintácticos dela lengua inglesa a los estudiantes cuya lengua materna era la croata-serbia. «En aquel entonces, de hecho, realizaba el análisis contrastivo entre el croata-serbio y el inglés, aunque no utilizaba este término». De acuerdo con Filipović (1968a: 1-5) y Santos Gargallo (1993: 27), el término Lingüística contrastiva fue acuñado por el lingüista norteamericano Trager en 1949 en su obra The Field of Linguistics:

But once the linguist has arrived at descriptive grammars of at least two languages, he may compare them. When his activities are concerned with showing the structural differences and resemblances of the systems, he is doing CONTRASTIVE LINGUISTICS (Trager, 1949: 6).

Filipović (1968a: 1-5) llega a dos conclusiones con respecto a la distinción entre los términos comparar y contrastar. La primera conclusión es que la Lingüística comparativa trata lenguas genéticamente relacionadas; el objetivo del Análisis comparativo es investigar relaciones genéticas entre estas lenguas. La segunda conclusión atañe al contrastivismo: la Lingüística contrastiva estudia lenguas genéticamente no relacionadas; el objetivo del Análisis contrastivo es contraponer segmentos correspondientes a lenguas genéticamente distintas.

\subsection{Período moderno}

El período moderno, siguiendo la clasificación de Đorđević (2004), podría considerarse el tiempo que abarca desde la publicación de la obra Contrastive Structure Series ${ }^{5}$ en 1965, y que perdura hasta el presente.

$5 \quad$ Es una colección de diez estudios publicada como resultado del primer proyecto dedicado a estudios contrastivos, realizado en el Centro de Lingüística Aplicada de Washington entre 1962 y 1965. 
Lo caracteriza un amplio número de proyectos contrastivos, la expansión de estudios teóricos, la coordinación internacional entre los expertos y una proliferación continua de trabajos y publicaciones. Asimismo, se observan las primeras críticas dirigidas a algunas formulaciones teóricas originadas durante el período clásico. Según afirma Đorđević (2004), esto es normal ya que justifica el desarrollo de una disciplina científica $y$, al mismo tiempo, evidencia un gran interés por los estudios contrastivos.

Por lo que respecta a la cronología de las actividades, es necesario, según Santos Gargallo (1993: 43) «bifurcar geográficamente la historia de la Lingüística contrastiva, considerando por separado las actividades de los EE. UU. y Europa, respectivamente. Veremos cómo en el momento en que la Lingüística Contrastiva decae en los EE. UU., como resultado de las duras críticas a las que se ve sometida, resurge en Europa».

En los Estados Unidos, en la Universidad de Georgetown, en Washington, se organiza en 1968 una conferencia sobre la Lingüística contrastiva. Siguiendo a Santos Gargallo (1993: 44), «esta conferencia introdujo el Análisis de Errores como réplica al Análisis Contrastivo, modelo que surge para cubrir las deficiencias y carencias de los estudios contrastivos. Sin embargo, como veremos más tarde, el Análisis de Errores no excluye el Análisis Contrastivo». El artículo de Corder, "The Significance of Learner's Errors", publicado unos meses antes, en noviembre de 1967, se considera oficialmente el inicio del modelo de Análisis de errores. Cabe añadir, y siguiendo el orden cronológico, que en 1971 se organiza en Hawái la Conferencia del Pacífico sobre Análisis Contrastivo y Universales Lingüísticos que produce, según Santos Gargallo (1993: 44), la revisión y reevaluación del Análisis contrastivo.

En lo referente al espacio europeo, ha surgido una variedad de conferencias, seminarios y proyectos contrastivos. Siguiendo a Đorđević (2004: 16-18), en 1970 se celebró la conferencia de Zagreb, en 1975 la de Bucarest, en 1978 hubo dos encuentros en las ciudades alemanas de Trier y Saarbrücken, en 1980 se preparó el encuentro internacional de Polonia y en 1982 se convocó otro en Finlandia. Asimismo, se celebran seminarios anuales en Polonia. En Novi Sad se han organizado simposios sobre estudios contrastivos cada tres años. Con respecto a los proyectos contrastivos, cabe mencionar los realizados en Bélgica, España, Estonia, Finlandia, 
los Países Bajos, Polonia, Rumanía, Suecia y Yugoslavia. Uno de los primeros y más significativos proyectos contrastivos fue el Proyecto Contrastivo Yugoslavo Serbo-Croata Inglés, YSCECP, iniciado en 1968 y terminado en 1986, bajo la dirección del profesor Filipović (1968c).

\section{Críticas al análisis contrastivo}

Las primeras críticas al Análisis contrastivo aparecieron en el año 1968, durante la conferencia sobre la Lingüística contrastiva organizada por la Universidad de Georgetown, en Washington, como hemos indicado en 1.3., refiriéndose tanto al plano teórico como al práctico. Asimismo, las críticas se manifestaron en el Congreso de la Federación Internacional de Profesores de Lenguas Vivas (FIPLV), celebrado en Zagreb, en abril de 1968. Siguiendo a Penadés Martínez (1999: 7): «[...] unas han sido planteadas como fruto de una reflexión teórica, las Ilamadas paradoja gramatical, paradoja semántica y paradoja pedagógica [...], y otras [son] de orden más bien empírico: diferentes investigaciones sobre la validez predictiva del análisis contrastivo demostraron, entre otras particularidades, que allí donde no debía producirse un error, por la similitud entre la L1 y la L2, los aprendices de L2 se equivocaban y, por el contrario, estructuras diferentes entre ambas lenguas eran asimiladas sin ninguna dificultad».

\subsection{Críticas hacia los aspectos teóricos}

En lo que concierne a los tres aspectos teóricos que causan problemas en los estudios contrastivos, la paradoja gramatical, la semántica y la pedagógica, siguiendo a Krzeszowski (1990: 1-8), la paradoja gramatical consiste en el hecho de que las lenguas pueden compararse en la medida en que sean similares. Según el esquema de Krzeszowski (1990: 5), que a continuación presentamos, cuanto más se diferencian dos lenguas, menos se pueden comparar, ya que no tienen elementos para contrastar. Esto conduce a la paradoja, siendo el fundamento de los estudios contrastivos que la diferencia es la que provoca dificultades y, por tanto, las diferencias son las que se investigan. 


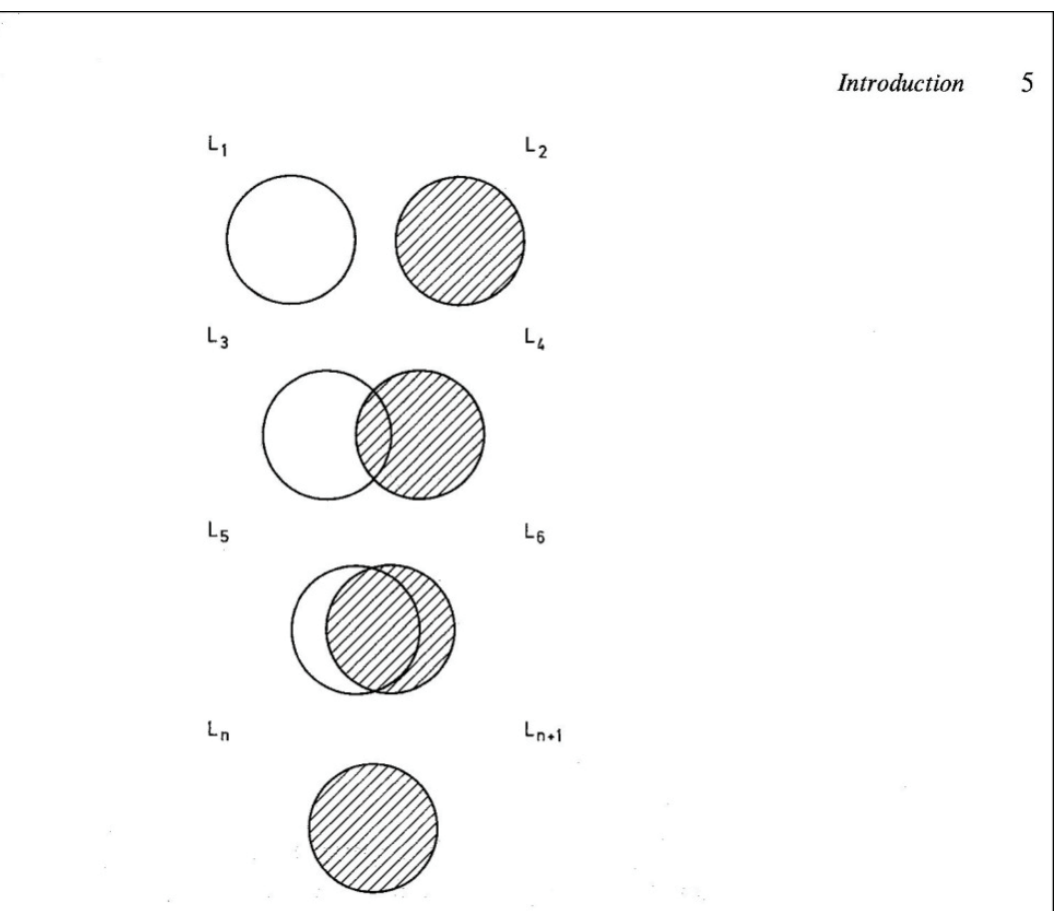

Figure 1. Languages in a cline ranging from those with nothing in common to those that are identical.

Gráfico de Krzeszowski (1990: 5): El diagrama presenta las lenguas desde arriba hacia abajo distinguiendo entre las que no tienen nada en común y las que son idénticas.

En lo referido a la paradoja semántica, Krzeszowski (1990: 6-7) parte de la hipótesis de que todos los enunciados de todas las lenguas derivan de una base semántica universal. Se supone que existe una representación semántica universal, libre de categorías específicas de una lengua, de la que todos los enunciados de todas las lenguas se derivan a través de las categorías y las normas sintácticas propias de un idioma particular. Por ello, la hipótesis sobre la igualdad de las representaciones semánticas, derivadas de los enunciados equivalentes, lleva a la paradoja semántica, puesto que lo que es idéntico no está sujeto a la comparación, mientras que lo que es diferente no es comparable.

La paradoja pedagógica consiste en el hecho de que las características de una lengua, consideradas triviales y poco interesantes por los lin- 
güistas teóricos, son, en efecto, de suma importancia pedagógica. Krzeszowski (1990: 8) parte de la gramática universal, afirmando que lo que es universal debe ser familiar para todos los estudiantes de una lengua extranjera debido a que los aprendientes parten de su lengua materna. Sin embargo, hay que tomar en consideración que los estudiantes deben aprender lo que no les resulta familiar, esto es, son elementos no comparables que forman parte de una información específica e idiosincrática que, por lo general, es ignorada por los estudios contrastivos.

\subsection{Críticas hacia los aspectos empíricos, inicios del Análisis de errores}

Algunas de las críticas dirigidas hacia el Análisis contrastivo y su función aplicativa dentro de la enseñanza de lenguas extranjeras se deben, según Riđanović (1978) ${ }^{6}$, a un cierto malentendido entre los lingüistas y los pedagogos dedicados al proceso de docencia. Los últimos objetaban que:

- $\quad$ el Análisis contrastivo es de disputable aplicabilidad pedagógica, ya que los problemas derivados de la lengua materna no representan la única causa de los errores;

- un profesor con experiencia docente sabe dónde cometen errores sus alumnos sin acudir al Análisis contrastivo;

- las predicciones del Análisis contrastivo no son siempre válidas;

- el Análisis contrastivo introduce en el aula la lengua materna de manera exagerada, por lo que complica el proceso de aprendizaje.

Riđanović sostiene que el malentendido reside en el hecho de que los lingüistas nunca han insistido en que el Análisis contrastivo pueda predecir todos los errores y, asimismo, lo justifica con su propia investigación docente, donde los errores contrastivos sumaban un número comprendido entre el $65 \%$ y $80 \%$ del total de los cometidos. A continuación, admite que un profesor con experiencia puede discernir un cierto número de errores contrastivos pero no puede identificarlos todos; luego, sostiene que los lingüistas nunca han dicho que todas las predicciones del

6 Midhat Riđanović fue uno de los investigadores que tomaron parte en el Proyecto Contrastivo Yugoslavo Serbo-Croata Inglés, YSCECP. 
Análisis contrastivo sean válidas en un $100 \%$. En lo que atañe a la última crítica, la relacionada con el papel de la lengua materna en el aula, Riđanović se refiere a lo que se ha convertido en lema de la docencia de los años setenta: "La lengua materna se puede excluir del aula pero nunca puede ser expulsada de la cabeza del estudiante».

No obstante, y como ya hemos indicado en 1.3., el artículo de Corder, "The Significance of Learner's Errors", publicado en noviembre de 1967, se considera el inicio de un nuevo modelo de la Lingüística contrastiva, el modelo del Análisis de errores. Este modelo ha sido aceptado por la mayoría de los lingüistas como una herramienta complementaria en los estudios contrastivos.

Uno de los primeros proyectos que reunió los dos métodos, el Análisis contrastivo y el Análisis de errores, fue el Proyecto Contrastivo Yugoslavo Serbo-Croata Inglés, YSCECP, iniciado en 1968 y terminado en 1986 bajo la dirección del profesor Filipović (1968c). El Proyecto fue organizado por el Instituto de Lingüística de Zagreb. Filipović fue uno de los fundadores de este Instituto en 1960, ejerciendo el cargo de su director entre 1963 y 1983 . Además de la sede administrativa del Proyecto en Zagreb, se constituyeron sendos centros de trabajo en Zagreb y Belgrado. Asimismo, el centro de trabajo de Zagreb incluía grupos de trabajo en Zadar, mientras que el centro de Belgrado contaba con grupos de trabajo ubicados en las ciudades de Sarajevo y Novi Sad. Siguiendo las referencias bibliográficas de Đorđević (2004), durante los dieciocho años de actividad del Proyecto se publicaron dos volúmenes sobre el YSCECP, en 1975 y 1978, diez libros de trabajo de la serie $A$. Reports, seis libros de la serie $B$. Studies, más cuatro libros de la serie New Studies.

Según Filipović (1975: 6), la experiencia del proyecto YSCECP ha demostrado desde sus inicios que los proyectos contrastivos aplicados no pueden quedarse solo en el análisis contrastivo, sino que deben llevar a cabo, en el transcurso del trabajo y de manera paralela, un análisis de errores. Los resultados de este análisis de errores pueden ser de gran utilidad tanto para la identificación de los problemas más generales como para un estudio sistemático de varias preguntas que surjan cuando se comparen dos sistemas lingüísticos. O sea, los dos análisis son comple- 
mentarios, esto es, cada uno por sí mismo es bueno pero combinados son más útiles y ofrecen una información necesaria para el mayor desarrollo y mejor aplicación de los materiales pedagógicos.

En cuanto a qué análisis llevar a cabo primero, el contrastivo o el de errores, Riđanović (1978) propone considerarlo desde dos posiciones. Por un lado, si el objetivo del estudio es la confección de materiales pedagógicos, es preferible realizar el Análisis de errores antes que el Análisis contrastivo. Por otro lado, si los objetivos de los estudios contrastivos van más allá de los meramente pedagógicos, lo que es el caso de la mayoría de los proyectos contrastivos, es necesario que el Análisis contrastivo preceda al Análisis de errores.

\section{Aspectos positivos del análisis contrastivo}

Lo que fue el objetivo inicial del Análisis contrastivo, predecir y explicar los errores causados por la interferencia de las estructuras de la lengua materna en la lengua de aprendizaje, se ha convertido, en el transcurso de los años de investigación, en un método contrastivo-analítico con resultados valiosos tanto en el campo de la lingüística teórica como en el de la aplicada.

Dentro de su marco de aplicación, el Análisis contrastivo dio un paso importante al concienciar al alumno de ser el protagonista de la clase y, por consiguiente, indujo la preocupación por el proceso de aprendizaje, según indica Santos Gargallo (1993: 67), temas que actualmente forman parte importante de las directrices establecidas por el Marco común europeo de referencia para las lenguas: enseñanza, aprendizaje, evaluación (MCER), publicado en 2002, y el Plan curricular del Instituto Cervantes (PCIC), publicado en 2006 con el fin de especificar los objetivos y contenidos de la enseñanza del español. La elaboración de gramáticas contrastivas de pares de lenguas, la materna con la lengua meta, ha proporcionado explícitamente las particularidades que distinguen una lengua de la otra y ha puesto de manifiesto sus similitudes y diferencias respectivas. Además, el Análisis contrastivo se ha convertido en un apoyo cien- 
tífico para los profesores. Cabe añadir que los estudios contrastivos han logrado un carácter académico. En este sentido, la Facultad de Filología de Belgrado ha impartido cursos de Lingüística contrastiva desde 1979, en la Cátedra de Estudios Anglísticos y, a partir de los años 80, en el Departamento de Lengua y Literatura Española, actualmente el Departamento de Estudios Ibéricos. Resumimos las aportaciones del Análisis contrastivo dentro de su marco de aplicación:

- el alumno es protagonista en la adquisición de una lengua extranjera

- se toma en consideración el proceso de aprendizaje

- elaboración de gramáticas contrastivas

- apoyo científico de los profesores

- conexión con la psicolingüística

- consideración de la interlengua como categoría psicolingüística

- conexión con la sociolingúística

- útil para la variedad lingüística

En lo que concierne al papel del Análisis contrastivo dentro del marco teórico, su actividad más importante ha sido dirigida hacia los estudios de Lingüística descriptiva y lingüística teórica general. Siguiendo a Ferguson (1971: 233-234), dos objetivos fundamentales de la lingüística son:

1. El desarrollo de una teoría general sobre el lenguaje humano

2. El desarrollo de los procedimientos que proporcionen una descripción completa de cualquier lengua particular

Ferguson (1971: 233-234) indica que para conseguir ambos objetivos en algún momento será necesario comparar detalladamente gramáticas de diversas lenguas con el fin de descubrir las similitudes subyacentes y distinguir estas de las similitudes y diferencias superficiales que perfilan el carácter distintivo de cada lengua. Asimismo, es posible describir una lengua en particular, o sea, describir su gramática, sin hacer referencia explícita a otras lenguas. No obstante, cuando el propósito de esta descripción sea mostrar las formas en que una lengua difiere de otras, será necesario acudir al Análisis contrastivo. 


\section{Revitalización del análisis contrastivo}

En la actualidad, la Lingüística contrastiva, o más concretamente, el Análisis contrastivo, constituye a la par que una rama de la Lingüística general, un soporte básico de la Lingüística aplicada. Desde la perspectiva de la Lingüística general, los resultados del Análisis contrastivo se utilizan para numerosas investigaciones útiles, sobre todo para la Tipología lingüística y el establecimiento de universales lingüísticos. Desde una perspectiva aplicada, la Lingüística contrastiva supone una herramienta práctica para la enseñanza de lenguas extranjeras, para la traducción o para la investigación sobre el bilingüismo.

\subsection{Aspectos teóricos. La Lingüística tipológica y universalista}

En lo que concierne a la tipología lingüística y los universales, siguiendo a Moreno Cabrera (1997: 13), se trata de «aquella rama de la ciencia del lenguaje que se ocupa de estudiar las semejanzas y las diferencias entre las lenguas humanas, siendo la investigación tipológica la que intenta mostrar que las formas en las que varían las lenguas o familias lingüísticas siguen unos patrones definibles que se pueden determinar con mayor o menor precisión». Es decir, aunque parezca que hay una diversidad sin límites entre las lenguas del mundo, existe una serie limitada de principios y patrones estructurales generalizados que se repiten en las diversas lenguas del mundo. A continuación, presentamos unos parámetros concretos según el contraste realizado por Moreno Cabrera (2010) respecto al turco y nuestra competencia en lo que se refiere al serbio.

El patrón verbo + objeto (VO) / objeto + verbo (OV)

\begin{tabular}{|l|c|l|}
\hline \multicolumn{3}{|c|}{ Patrón VO / OV } \\
\hline español & VO & Bebemos té ('Pijemo čaj') \\
\hline turco & OV & Çay içiyoruz ('Té bebemos') \\
\hline serbio & VO & Pijemo čaj ('Bebemos té') \\
\hline
\end{tabular}

Tabla de parámetros tipológicos 
Este patrón se identifica por el orden en que cada una de las lenguas observadas presenta el verbo (V) o el objeto directo (O), según el cual la posición no marcada o normal del verbo puede ser anterior o posterior a la del objeto directo. Este rasgo suele ir unido a otras características morfosintácticas que nos permiten suponer lo que vamos a encontrar en una determinada lengua. Así, podemos decir que el español es una lengua VO, Bebemos té, mientras que el turco es OV, 'Té bebemos'. En este sentido el serbio es una lengua VO, Pijemo čaj 'Bebemos té'.

Según esta constatación, el parámetro VO / OV nos indica que otros elementos de la gramática seguirán el modelo VO, en el que el rector (V) va antes que lo regido (O), o seguirán el modelo OV en el que lo regido (O) va antes que el rector (V). Por ejemplo:

- Los determinantes posesivos, posesivo + nombre / nombre + posesivo:

En español los determinantes posesivos se colocan delante del sustantivo, mi casa, pero en turco se colocan detrás, 'casa mi'. En serbio se colocan igual que en español, moja kuća 'mi casa'.

- La posición del adjetivo especificativo, nombre + adjetivo / adjetivo + nombre:

En español el adjetivo especificativo siempre va detrás del nombre, gato negro, mientras que en turco el nombre se pone obligatoriamente después del adjetivo, 'negro gato'. En serbio, esta es la única posición en que difiere del español y es igual al turco: crni mačak 'negro gato'.

- Núcleo + sub. adjetiva / sub. adjetiva + núcleo:

En español, la oración subordinada adjetiva se coloca después del sustantivo al que modifica: El hombre que ha hablado, pero en turco ocurre exactamente lo contrario: 'Habla-do es-que hombre'. En serbio, igual que en español, la subordinada adjetiva se coloca después del sustantivo, Čovek koji je govorio 'El hombre que ha hablado'.

De este contraste tipológico entre las tres lenguas, el español, el turco y el serbio, podemos concluir que el español es una lengua VO y sigue este patrón, el turco es una lengua OV y, asimismo, sigue su patrón tipológico de OV. Sin embargo, el serbio, aunque es una lengua VO, no 
siempre sigue este patrón tipológico, lo que justifica la posición de los lingüistas que están a favor del Análisis de errores, dado que las similitudes entre las lenguas no siempre facilitan el aprendizaje de la lengua extranjera sino que pueden llevar al error.

\subsection{Aspectos prácticos. Pragmática. Estrategias}

La Pragmática está estrechamente vinculada con los aspectos prácticos de la comunicación, centrados principalmente en las representaciones del habla, y es ahí donde se organizan las estrategias de comunicación. Lo cierto es que, siguiendo a Cenoz Iragui (1999), la adquisición de la competencia pragmática puede presentar dificultades ya que está relacionada con patrones sociales y culturales diferentes a los que tiene el aprendiente. En este sentido, un análisis contrastivo puede indicar las similitudes o las diferencias que existen a nivel pragmático y evitar las transferencias negativas de la lengua nativa a la extranjera, cuyo resultado es la producción de enunciados no apropiados. Asimismo, siguiendo un ejemplo de Riđanović (1978: 88), es posible que un contenido lingüístico de una lengua varíe en función de comportamiento extralingüístico o pragmático habitual dentro del ambiente de la lengua y cultura extranjeras. En particular, el autor ofrece un contraste pragmático en el uso de la respuesta a la expresión equivalente a la española gracias observándolo en varias lenguas: en el entorno lingüístico y cultural serbio y alemán es prácticamente inadmisible no responder a un hvala serbio o a un danke alemán con un molim serbio o con un bitte alemán. Sin embargo, el thank you inglés, en la mayoría de los casos, se queda sin una respuesta verbal explícita.

En lo que concierne a las estrategias, según la clasificación de García Santa-Cecilia (1995), el aprendiente parte de dos tipos de estrategias: las estrategias de comunicación, que le permiten desenvolverse en situaciones comunicativas; y las estrategias de aprendizaje, que le ayudan a controlar y desarrollar su propio aprendizaje de forma más eficaz. Estas dos estrategias gozan de mutua influencia aunque parezca que las estrategias de aprendizaje, que son de naturaleza intencional, no intervienen en el desarrollo de las estrategias de comunicación, que exigen una respuesta inmediata. Sin embargo, un análisis más profundo ha permitido entender 
que las estrategias de aprendizaje tienen sentido solo en cuanto son aplicadas en la realización de tareas comunicativas.

Las clasificaciones de las estrategias han provocado numerosas polémicas a la hora de definirlas; sin embargo, como señala Ciesielkiewicz (2010), entre las tipologías más claras, coherentes e influyentes se encuentran las de Oxford y las de O'Malley et al. Siguiendo sus clasificaciones es posible establecer la relación entre la Lingüística contrastiva y las estrategias y deducir que la Lingüística contrastiva facilita la comprensión y la memorización en el proceso de aprendizaje y adquisición de lenguas extranjeras. Según la tipología de O'Malley, la Lingüística contrastiva sirve de estrategia cognitiva mientras que, para Oxford, la Lingüística contrastiva da cabida a estrategias directas, puesto que ayuda a desarrollar la memoria y a mejorar la comprensión y producción oral y escrita por medio de un análisis deductivo y de las asociaciones con el conocimiento previo7. Por esto, la lengua materna es el instrumento fundamental del estudiante y le servirá de guía al aprender una lengua extranjera, o sea, el aprendiente dispone de una experiencia previa de aprendizaje lingüístico que le orientará en la construcción de su nuevo conocimiento lingüístico. De ahí que la aplicación de la Lingüística contrastiva, y de la gramática contrastiva como su resultado, constituya una estrategia fundamental de aprendizaje para los estudiantes de lenguas extranjeras. Además, la Lingüística contrastiva tiene importantes objetivos didácticos contextualizados en la elaboración del material didáctico que incluyen las gramáticas contrastivas.

Para demostrar que la Lingüística contrastiva ha estado y continúa estando vigente, Ciesielkiewicz (2010) ha llevado a cabo una investigación analizando cien manuales para la enseñanza o el aprendizaje de lenguas extranjeras.

En primer lugar, su investigación ha permitido determinar que el grado de aplicación de la gramática contrastiva en los manuales y libros de texto ha sido alto. En segundo lugar, ha demostrado que no impor-

7 Una vez un español me preguntó cómo se dice cumpleaños en serbio. Cuando le contesté con la palabra compuesta serbia rođendan 'el día de nacimiento', la persona la relacionó inmediatamente con el vocablo inglés birthday 'el día de nacimiento' para facilitar su compresión y memorización. 
taba cuál fuera la distancia entre la lengua materna y la lengua extranjera para llevar a cabo contrastes. Por ejemplo, se analizaban el ruso y el español, se contrastaba el polaco con el turco o el lituano, y el español con el húngaro o el japonés. En el tercer paso, contó con los niveles de enseñanza, lo que le permitió descubrir que la gramática contrastiva se utilizaba en manuales de cualquier nivel de enseñanza: básico, intermedio o avanzado. Finalmente, la autora observó las fechas de publicación de los manuales y concluyó que la Lingüística contrastiva nunca ha dejado de utilizarse a pesar de las fuertes críticas de las que ha sido objeto.

Asimismo, la gramática contrastiva, aplicada a los rasgos que presentan más dificultades, puede ser un excelente medio de aprendizaje y autocorrección; además, se puede destinar a los aprendientes que tienen muy poco conocimiento de gramática, dado que los comentarios contrastivos permiten explicar los conceptos sin usar excesivamente el metalenguaje.

La investigación de Ciesielkiewicz (2010) ha puesto de relieve un elemento más: los comentarios de corte contrastivo se han aplicado al aspecto sociocultural o léxico tomando en consideración su valor comunicativo, de ahí que se hayan refutado las críticas dirigidas hacia el Análisis contrastivo de no atender al contexto sociocultural o comunicativo.

\section{El estado de la cuestión con respecto al serbio y al español}

Por lo que se refiere al material didáctico contrastivo elaborado en Serbia, en 2009 se publicó la Gramática de la lengua española para serbiohablantes con ejercicios, de Rajić y Marcos Blanco.

Asimismo, hay un conjunto de estudios contrastivos que han abordado los temas y problemas específicos de la gramática serbia con respecto a la española, así como varios aspectos de la traducción. Según Filipović (2010), las investigaciones contrastivas entre el serbio y el español se clasifican en cuatro campos teórico-metodológicos: investigaciones contrastivas teóricas, teórico-aplicadas, aplicadas e histórico-sociolingüísticas. Las investigaciones contrastivas teóricas están a cargo de las investigadoras Antonić, Bajić, Bajić-Nikolić con Alonzo Zarza, el investigador Belić y las investigadoras Pejović, Rajić y Veljković. Las investigacio- 
nes teórico-aplicadas y aplicadas son mérito de las autoras Bajić, Filipović, el tándem Jelena Filipović y César Díez Plaza, Pejović, Rajić y Zečević-Krneta. Por lo que respecta a las investigaciones histórico-sociolingüísticas, a este campo se han dedicado Filipović, Kuzmanović, Pejović, Pešić y Vučina-Simović. Las investigaciones contrastivas serbias pertenecen al período moderno de la Lingüística contrastiva e incluyen aportaciones de las ciencias actuales como, por ejemplo, principios de la teoría de adquisición de segundas lenguas, antropología cognitiva, análisis del discurso, sociolingüística crítica, etc. Asimismo, según indica Filipović (2010), «ofrecen una perspectiva interdisciplinaria que podría contribuir a un mejor entendimiento tanto de las estructuras lingüísticas de las lenguas contrastadas, como de los aspectos culturales, sociales, antropológicos, etc., de las comunidades de habla investigadas».

Los estudios que indican los errores más frecuentes en las producciones escritas u orales de los estudiantes serbios son de escaso número. Una investigación de considerable importancia es la tesis de Santos Gargallo, defendida en 1992; sin embargo, esta investigación no incluye un análisis contrastivo de las lenguas española y serbocroata. En 2003, Filipović y Díez Plaza publican un artículo sobre los factores tipológicos relacionados con la adquisición de los sistemas fonológicos de las lenguas extranjeras. La investigación fue realizada basándose en el análisis de errores de un corpus de siete estudiantes cuya lengua materna es el español, mientras que su lengua de adquisición es el serbio. Otro trabajo de los autores referidos, Filipović y Díez Plaza, publicado en 2000, incluye ambos modelos de la Lingüística contrastiva, el modelo de Análisis de errores y el modelo de Análisis contrastivo. El tema de esta investigación es Los verbos de movimiento en español y en serbio: una aproximación al análisis semántico aplicado a la enseñanza de E/LE. Esta investigación trabaja sobre un corpus de estudiantes de español de la Facultad de Filología de Belgrado cuyos niveles de competencia se corresponden al primer, segundo, tercer y cuarto año de los estudios. Cabe añadir que en las Actas del I Congreso Internacional "Enseñanza de lenguas: teoría y práctica», celebrado en Belgrado en 2008, se encuentra un artículo (García González y Alonso Zarza, 2009) sobre el análisis de errores. Este artículo da cabida a un extenso estudio sobre los errores y sus causas. Sin embargo, 
no considera un análisis contrastivo entre la lengua española y la lengua serbia. Nos resulta relevante la observación que versa sobre los errores fosilizados, dado que indica que en primer lugar estos aparecen en el uso del artículo determinado, que es el tema de la tesis doctoral que estamos llevando a cabo. En el año 2013, la revista Colindancias (2013, núm. 4) publica el artículo «Análisis de errores en el aprendizaje del español por alumnos que tienen como lengua materna el serbio», elaborado por Krstić y Pištignjat, que atiende distintos fenómenos gramaticales. No obstante, este estudio no considera los errores en el uso del artículo ni realiza un análisis contrastivo.

\section{Justificación de la importancia del uso paralelo de los dos modelos}

Según hemos expuesto en 2.2., la experiencia del proyecto contrastivo dirigido por Filipović (1975) ha mostrado que los dos modelos de análisis son complementarios y, aunque cada uno por sí mismo es bueno, combinados son más útiles. Además, Riđanović (1978) realiza una sugerencia sobre el orden en el que se lleva a cabo el Análisis contrastivo y el Análisis de errores, indicando que es necesario que el Análisis contrastivo preceda al Análisis de errores si los objetivos de los estudios contrastivos van más allá de los exclusivamente pedagógicos.

Debido a estas aportaciones, así como gracias a nuestra propia experiencia investigadora, estamos llevando a cabo en nuestra tesis doctoral una investigación de las producciones escritas de aprendientes serbios, con el objetivo de analizar los errores en el uso del artículo determinado y ofrecer, previamente, un estudio contrastivo que incluya los mecanismos que utilizan las dos lenguas, serbio y español, para identificar la referencia del sustantivo. El título de nuestra tesis es Análisis de errores en el uso del artículo determinado en producciones escritas de aprendientes serbios de español como lengua extranjera. Este proyecto de investigación tiene como objetivo ampliar el análisis ya finalizado en nuestro trabajo final del Máster en formación de Profesores de Español, defendido en 2013, que lleva por título Análisis de los errores de concordancia 
en la expresión escrita de los aprendientes serbios de ELE. Es la investigación que en el presente artículo nos servirá para justificar la importancia del uso paralelo de los dos modelos de la Lingüística contrastiva. En dicho trabajo realizamos un análisis de los errores en la concordancia nominal y verbal existentes en 102 textos escritos por universitarios serbios, análisis que iba precedido por el correspondiente contraste de las lenguas española y serbia en relación con el fenómeno gramatical de la concordancia.

\subsection{Análisis contrastivo}

El primer paso ha sido realizar un análisis contrastivo entre el español y el serbio. Por lo tanto, primeramente hemos presentado el concepto de la concordancia española y, en segundo lugar, hemos trabajado sobre las particularidades de la concordancia serbia.

Dentro de la concordancia española, hemos investigado sobre distintas categorías de la concordancia nominal y verbal, así como sobre el papel del género, el número y la persona. Relacionamos la información con los niveles de competencia establecidos por el MCER y el PCIC y elaborados por Martí Sánchez, Penadés Martínez y Ruiz Martínez (2008) en su Gramática española por niveles, siempre teniendo en consideración los casos que habían aparecido en el corpus de las 102 redacciones. Hemos definido que el español distingue dos tipos de concordancia:

1. La concordancia nominal, esto es, la coincidencia de género y número que establece:

- el sustantivo con el artículo y otros determinantes (El maestro explicaba aquella lección.)

- $\quad$ el sustantivo con los adjetivos que lo acompañan (ojos melancólicos; tranquilas tardes veraniegas)

- el pronombre con su antecedente o consecuente (A tu hermano lo vi en el cine. Les di los libros a los estudiantes.)

- el sujeto con el atributo (Mi hija es trabajadora.)

- $\quad$ el sujeto con el complemento predicativo (Mi hijo llega cansado.)

- el sujeto con el participio del verbo de la pasiva perifrástica (Las obras fueron escritas.) 
2. La concordancia verbal, esto es, la coincidencia de número y persona, que es la que se establece entre el verbo y su sujeto (Ellos escriben rápido.).

Por lo que se refiere a la concordancia serbia, la hemos abordado en la medida de lo posible de manera recíproca a los conceptos españoles debido a que el serbio distingue la concordancia en el caso morfológico, además de la de género, número y persona. Asimismo, el serbio diferencia tres géneros, el femenino, el masculino y el neutro, mientras que el español no posee género neutro. Nos hemos basado en dos gramáticas serbias de referencia, la gramática de Stanojčić y Popović (2012) y la gramática de Klajn (2005). También hemos investigado sobre algunos casos específicos de concordancia serbia, como en particular la concordancia del género que puede ser a la vez gramatical y semántica dentro de un mismo grupo semántico u oración (On je velika pijanica. 'Él es grandiosa borracho'. Él es un gran borracho.); la concordancia del número en colectivos donde el determinante aparece en singular y el verbo en plural (Sva deca dolaze 8 . 'Toda niña vienen'. Todos los niños vienen.); o la concordancia de los numerales cardinales por la existencia en la lengua, y por lo tanto, en la mente de los serbios, del número paucal 'la pequeña cantidad', un concepto derivado de la antigua lengua eslava para designar los números entre dos y cuatro.

Hemos definido que el serbio, al igual que el español, distingue dos tipos de concordancia, la nominal y la verbal. La diferencia reside en que las reglas morfosintácticas serbias exigen:

1. En la concordancia nominal, que la coincidencia sea en caso morfológico además de en género y número.

2. En la concordancia verbal, que la coincidencia sea en género además de en número y persona en cuanto a los tiempos verbales compuestos. Sin embargo, en los tiempos verbales simples, la concordancia se presenta solo en número y persona, de la misma manera que en español. 


\subsection{Análisis de errores}

El análisis de errores ha sido la segunda etapa de nuestra investigación. En primer lugar, hemos realizado una indagación sobre los inicios del análisis de errores. En segundo lugar, hemos interpretado el contenido y el objetivo de la tesis de Santos Gargallo (1992): La enseñanza de segundas lenguas: Análisis de errores en la expresión escrita de los estudiantes de español cuya lengua nativa es el serbo-croata. En tercer lugar, hemos proseguido con las clasificaciones de los errores y su incidencia en el significante $y / o$ el significado de una palabra siguiendo la clasificación por niveles lingüísticos de Penadés Martínez (2003). Asimismo, hemos observado que los errores de concordancia se corresponden al significado instrumental de palabras y se manifiestan en sintagmas u oraciones. Posteriormente, hemos trabajado con el corpus de las 102 redacciones de los estudiantes serbios de la Universidad de Kragujevac. Son estudiantes del primer, segundo, tercer y cuarto año de la Facultad de Filología y Artes (FILUM), inscritos en el Departamento de Lengua española y literaturas hispánicas. El corpus fue realizado a finales del año lectivo 2010-2011 por Gorana Zečević Krneta, profesora ayudante del Departamento. Con el objetivo de analizar los errores, primero hemos establecido una clasificación gramatical de los errores (artículo determinado, artículo indeterminado, determinante demostrativo, determinante posesivo, determinante numeral cardinal, etc.) y de allí proseguimos con la investigación según la ocurrencia de los errores en relación con el género, el número y la persona. Hemos confeccionado de manera paralela gráficos para diversos ejemplos, ya que consideramos que la presentación visual ayuda a una mejor y más rápida percepción de los datos. Finalmente, llegamos a las conclusiones.

Nos resultó de gran utilidad la tesis doctoral de Santos Gargallo (1992), que trata de una manera académica y estructurada los tres modelos de investigación: el Análisis contrastivo, el Análisis de errores y la Interlengua. Su investigación tiene como propósito dos objetivos, según indica la autora en la introducción de su obra. El primer objetivo es la idea de que «el paso de un modelo al otro no significa el rechazo del anterior sino la superación del mismo en un esfuerzo científico común». El segundo 
objetivo es cubrir «la laguna existente», ya que en la época de su investigación «no existía ningún manual que desarrollara de manera vertebrada la evolución de esta parte de la lingüística». Santos Gargallo ha investigado la recurrencia de los errores en la expresión escrita de estudiantes de español cuya lengua nativa es el serbocroata según una clasificación basada en las categorías gramaticales que afectan a la morfología, la sintaxis y el léxico, presentando un cómputo cuantitativo de los siguientes contenidos lingüísticos: artículo determinado e indeterminado, tiempos de pasado, preposiciones, concordancia de género y número, ser/estar/ haber, pronombres, subjuntivo y, finalmente, léxico. En lo que concierne a la concordancia de género y número, la autora realiza dos investigaciones análogas, una para los errores del género y otra para los del número. También ha hecho referencia a la concordancia entre el sujeto y el atributo. La concordancia verbal no ha sido objeto de su investigación. Según Santos Gargallo (1992: 236), la causa de los errores de género reside en «un desconocimiento de las reglas básicas que regulan el género gramatical del sustantivo y cuyo aprendizaje constituye una de las primeras dificultades del estudiante extranjero». En cuanto a las causas de los errores del número, la autora dice: «creemos que más que a un desconocimiento de las reglas, se debe a una falta de cuidado en la redacción».

En relación con nuestro trabajo, la tesis de Santos Gargallo ha sido un incentivo para seguir investigando desde el punto en el que ella lo dejó. Aprovechamos en este sentido nuestro conocimiento nativo de la lengua serbia para establecer un contraste explícito con la española, cuestión no tratada en la tesis de esta autora. Por eso, decidimos entrar profundamente en el campo de la lingüística contrastiva y, en un segundo paso, optamos por una clasificación gramatical de los errores de concordancia.

Tras realizar la clasificación de los errores que aparecen en el Corpus de aprendices serbios de $E / L E$, en primer lugar, hemos observado que un gran número de errores están relacionados con el género de las palabras, mientras que un número inferior de errores se debe a la confusión con el número; concretamente, los errores de género suponen un 84,9 \% frente al $15,1 \%$ de número. Asimismo, hemos encontrado categorías en las que los errores de género aparecen en el $100 \%$ de los casos. Debido a que estas estructuras o combinaciones de palabras con el $100 \%$ de los 
errores necesitan reforzarse, las hemos presentado en el gráfico según el orden en el que aparecieron: artículo indeterminado + nombre, determinante numeral cardinal + nombre, determinante numeral ordinal + nombre, los determinantes todo, otro, alguno + nombre y nombre precedido de determinantes y con otros modificadores.

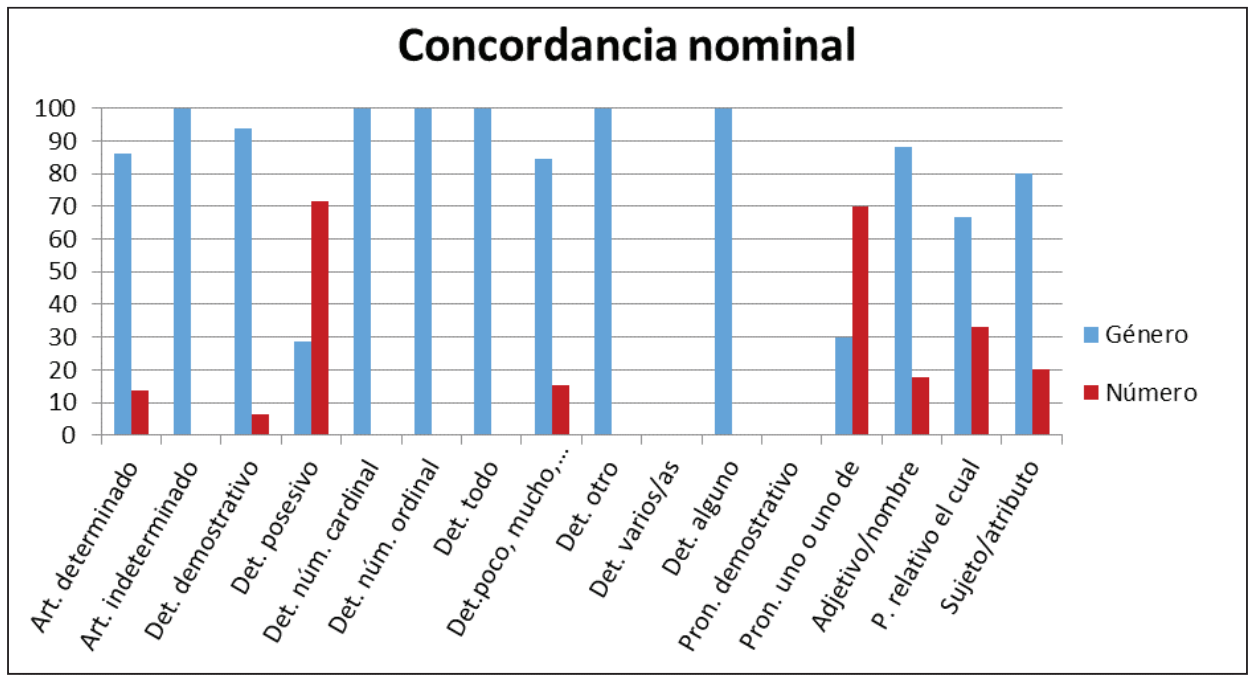

Relación de errores según las categorías gramaticales

Este tipo de errores confirma lo que ya apuntaba Gutiérrez Araus (2011: 119) sobre la concordancia, que es «una de las dificultades que presenta el español en el aprendizaje de estudiantes extranjeros cuyas lenguas no tienen los mismos morfemas nominales o no tienen morfemas nominales». También tiene influencia, según indicaba Pastor Cesteros (2001: 21), el hecho de que «a pesar de las numerosas excepciones, continúa leyéndose en manuales y gramáticas al uso que, en español, las palabras acabadas en - $a$ son femeninas y las acabadas en - 0 masculinas, lo cual provoca errores [...]».

En segundo lugar, hemos descubierto que las mismas palabras son objeto de errores de concordancia en distintas estructuras gramaticales (artículo determinado + nombre, artículo indeterminado + nombre, demostrativo + nombre, adjetivo + nombre, etc.) y están relacionadas con los errores de concordancia a lo largo de todo el corpus, es decir, a 
lo largo de los cuatro niveles de competencia que analizamos (A2, B1, B2, C1). Haciendo el conteo de estas palabras llegamos a la conclusión de que, de un total de 187 errores, el 44,1\%, se debe a solo ocho palabras: ciudad, país, gente, parte, viaje, costumbre, cosa y sueño.

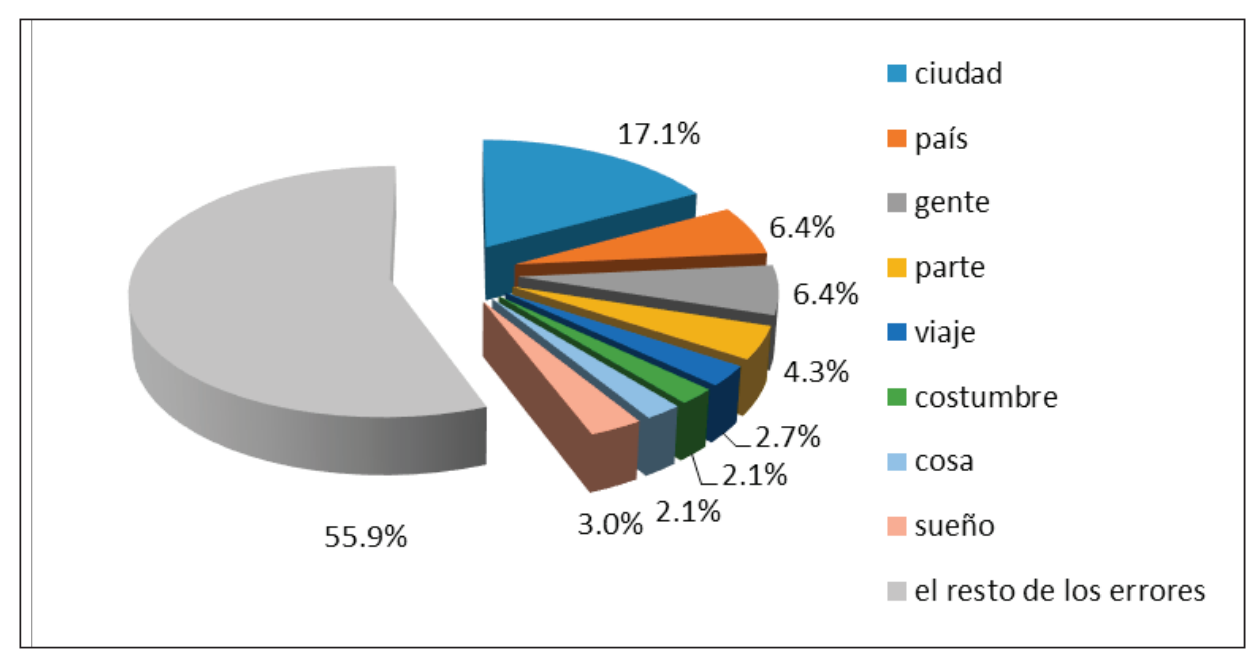

Gráfico de las palabras que causan error más frecuentemente

Debido a que previamente hemos hecho el análisis contrastivo de la concordancia del español con la del serbio, hemos percibido que los errores aparecen allí donde no deberían esperarse, puesto que en los casos donde se han encontrado las mencionadas palabras, las reglas gramaticales de las dos lenguas, el español y el serbio, son análogas. Por lo tanto, en estas situaciones la concordancia española no debería presentar problemas para los estudiantes serbios, debido a que está marcada en su propia gramática, que conocen bien. Dada esta situación, decidimos investigar la recurrencia de los errores de concordancia relacionados con cada palabra en particular, por un lado, desde un punto de vista empírico y, por el otro, desde el punto de vista del hablante nativo serbio, para tratar de ofrecer una explicación sobre los mecanismos psicológicos (de interferencia o transferencia negativa) que entran en juego al concordar dichas palabras.

La palabra ciudad constituye un $17,1 \%$ del total de los errores y aparece en la mayoría de las distinciones de errores que hemos expuesto. El problema reside en el género. Debido al hecho de que la palabra serbia 
grad 'ciudad' es masculina, el estudiante mantiene el concepto de género masculino aunque habla español, precisamente porque esta palabra es muy corriente y, por lo tanto, está fuertemente marcada en la mente del alumno. Su influencia es tan grande que se mantiene hasta los niveles más altos de la competencia del español. Asimismo, contribuye al error el hecho de que la palabra española no acaba en - $a$.

La misma situación presentan las palabras país, parte, viaje y costumbre. Aunque en el tercer año lectivo los errores disminuyen, siguen presentes hasta los niveles más altos de la competencia de ELE. Además, la palabra costumbre había sido erradicada en el tercer año, pero aparece de nuevo en el cuarto, evidentemente debido a que la marca de género masculino de la palabra serbia persiste en la mente del estudiante. Como ya hemos indicado en el ejemplo anterior, contribuye al error el hecho de que las palabras españolas (parte, costumbre) no acaban en - $a$, ni tampoco las masculinas (país, viaje) terminan en -o.

En consecuencia, podemos deducir que allí donde no deberían aparecer errores debido a la igualdad de las reglas de concordancia entre dos lenguas, estos aparecen por la transferencia del comportamiento gramatical de las palabras de la lengua materna a la lengua meta, razón por la cual suponemos que se pone en marcha un mecanismo psicológico.

Por otro lado, el sustantivo colectivo ${ }^{9}$ gente es una palabra que sí puede provocar confusión en aprendientes serbios a la hora de concordarla, tanto en género como en número y tanto en la concordancia nominal como en la verbal. En este caso, la Lingüística contrastiva nos ayuda a prever el problema. Además de la transferencia del género (que en serbio es masculino), la causa de los errores reside también en la transferencia de las reglas gramaticales de concordancia del serbio al español. En español, este vocablo es un nombre colectivo y concuerda en singular. Si el atributo se encuentra lejos, se admite plural. En serbio, la palabra ljudi 'gente' es un plural supletivo del sustantivo masculino singular čovek 'hombre'. Por lo tanto, no se trata de un colectivo sino solo de una forma en plural, un pluralia tantum. Debido a esto, concuerda siempre en plural. rales léxicos, en el sentido de que denota un conjunto de individuos. 


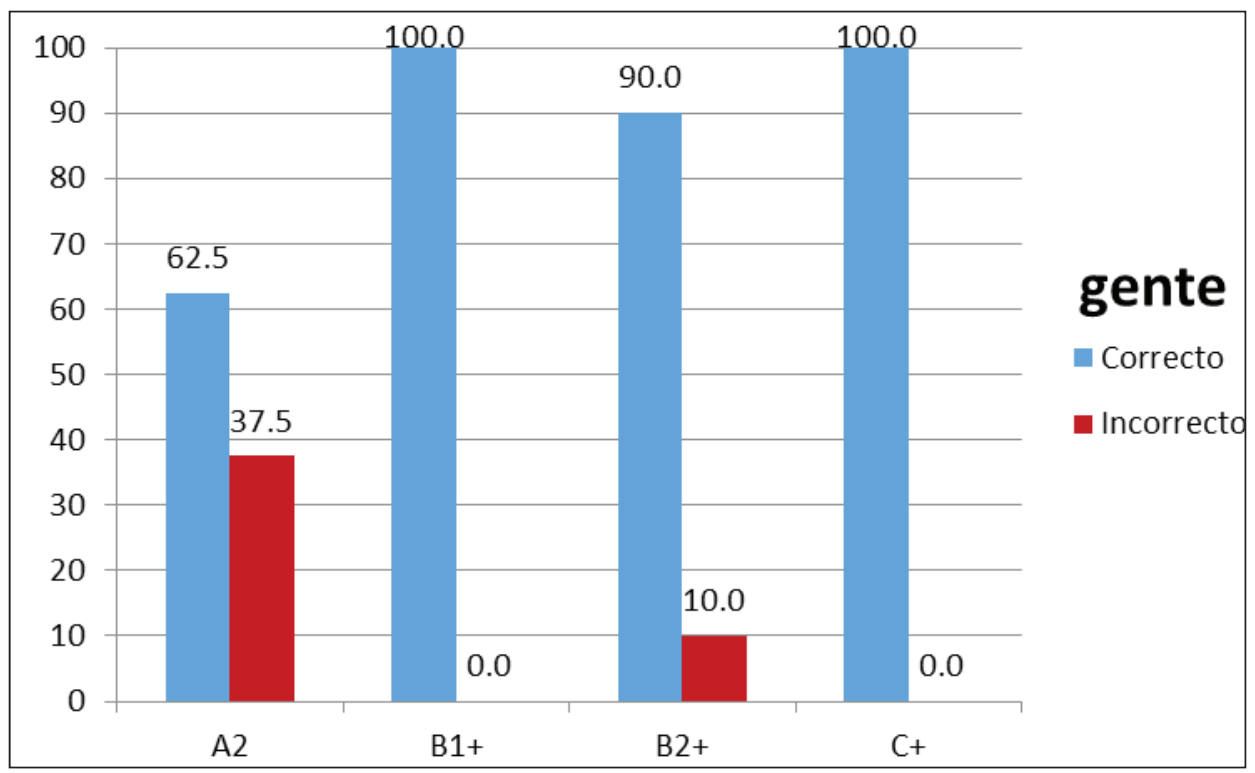

Gráfico de errores con la palabra gente 'ljudi'

En el gráfico se puede ver reflejado el trabajo explícito de los docentes para erradicar errores de concordancia con gente con óptimos resultados ya en el segundo año. El problema con gente apareció en algunos casos de nuevo en el tercer año, pero desaparece definitivamente en el cuarto curso.

\section{Conclusiones}

Como consecuencia del análisis percibimos que la causa de una gran parte de los errores cometidos reside más en el distinto comportamiento gramatical de sustantivos concretos en español y en serbio que en el desconocimiento de las reglas gramaticales del español, sobre todo porque esas reglas muchas veces coinciden con las serbias. En cuanto al género, el mayor problema lo presentan palabras cotidianas, frecuentes $y$, por lo tanto, muy fuertemente marcadas en la mente del estudiante, lo que determina el género con que el aprendiente la concordará en español. Debido a eso, la causa de los errores reside en la transferencia del comportamiento gramatical de las unidades de una lengua a las de la otra, lo que supone que entra en juego un mecanismo psicológico. 
En cuanto a las reglas de concordancia, el problema está en su semejanza pero no igualdad con las serbias. Un buen ejemplo son las reglas de concordancia con el colectivo. Opinamos que si no existiesen reglas similares sino completamente distintas, esas no serían causa de los errores de concordancia. En tal caso, la única tarea sería aprender reglas gramaticales, como ha señalado Santos Gargallo (1992: 236). Sin embargo, la existencia de reglas similares causa la confusión y esta se mantiene hasta los niveles más altos de la competencia de español.

La presente investigación ha justificado:

a) la fiabilidad de las conclusiones obtenidas por Corder (1967) de que la Lingüística contrastiva no es suficiente para encontrar las causas de los errores porque no siempre aparecen allí donde se prevén y aparecen allí donde no se han previsto;

b) la postura de Santos Gargallo (1992) de que los tres pilares de la investigación dentro de la Lingüística aplicada son la Lingüística contrastiva, el Análisis de errores y la Interlengua;

c) el planteamiento de la autora de esta investigación de que un mecanismo psicológico puede ser un factor adyacente a las causas de los errores de concordancia;

d) que la Lingüística contrastiva junto con sus dos métodos, el Análisis contrastivo y el Análisis de errores, ha justificado su vitalidad tanto en su marco teórico como en su marco de aplicación.

\section{BIBLIOGRAFÍA}

Andrews, Carol. The Rosetta Stone. London: British Museum Publications Ltd., 1987. Print.

Bloomfield, Leonard. Language. New York: Henry Holt and Company, Inc., 1956. Print.

Cenoz Iragui, Jasone. "Pragmática contrastiva: comparación de la producción de actos de habla en inglés y español". Estudios de Lingüística contrastiva (Actas del I Congreso de Lingüística Contrastiva. Lenguas y culturas, Santiago de Compostela, 21-23 de mayo de 1998). 
Santiago de Compostela: Universidad de Santiago de Compostela, 1999. 151-158. Impreso.

Ciesielkiewicz, Monika. "El resurgir de la lingüística contrastiva en manuales de lenguas extranjeras". Actas del IX Congreso Internacional de Lingüística General, 21-23 de junio de 2010, Universidad de Valladolid, Actas del Congreso. Valladolid: Universidad de Valladolid, 2010. 543-559. Impreso.

Corder, Stephen Pit. "The Significance of Learner's Errors". International Review of Applied Linguistics in Language Teaching, Vol. 5, No. 4 (1967): 161-170. Print.

Đorđević, Radmila. Uvod u kontrstiranje jezika. Beograd: Filološki fakultet Univerziteta u Beogradu, 2004. Štampano.

Ferguson, Charles Albert. "Contrastive Analysis and Language Development". Language Structure and Language Use: Essays by Charles A. Ferguson. Stanford, California: Stanford University Press1971. 233-234. Print.

Fernández González, Jesús. "El análisis contrastivo: historia y crítica”. Lynx. Documentos de trabajo, Vol. 1 (1995): 1-20. Impreso.

Filipović, Jelena. "Investigaciones contrastivas de las lenguas serbia y española". Ivan Klajn y Predrag Piper (eds.). Conttrastive Studies of Serbian Language: Trends and Results, Vol. 1. Beograd: SANU, 2010. 352-375. Štampano.

Filipović, Jelena y César Díez Plaza. "Los verbos de movimiento en español y en serbio: una aproximación al análisis semántico aplicado a la enseñanza de E/LE". Filološki pregled, Vol. XXVII, No. 1 (2000): 121132. Štampano.

Filipović, Jelena y César Díez Plaza. „Struktura sloga y srpskom kao L2: Tipološki faktori koji utiču na usvajanje fonoloških sistema drugih/ stranih jezika". Govor i jezik / Speech and Language. Beograd: Institut za eksperimentalnu fonetiku i patologiju govora / Institute for Experimental Phonetics and Speech Pathology, 2003. 137-147. Štampano.

Filipović, Rudolf. „Zašto kontrastivna analiza?“. Živi jezici, Vol. X, No. 1-4 (1968a): 1-5. Štampano.

Filipović, Rudolf. „Uloga kontrastivne analize u lingvističkom istraživanju“. Filološki pregled, Vol. III-IV. (1968b): 1-10. Štampano.

Filipović, Rudolf. Organizacija i zadaci projekta / The Organization and Objectives of the Project. Zagreb: Institut za lingvistiku Filozofskog fakulteta Sveučilišta u Zagrebu, Jugoslovenski projekat za kontrastivnu analizu srpskohrvatskog i engleskog jezika / The Yugoslav 
Contrastive Analysis Project. Serbo-Croatian and English (YSCECP), 1968c. Štampano.

Fries, Charles Carpenter. Teaching and learning English as a foreign language. Ann Arbor: The University of Michigan Press, 1974. Print.

García González, Javier y M. Ángeles Alonso Zarza. "Análisis de errores en español de serbohablantes". Zbornik radova, I Međunarodni kongres. Jezik struke: teorija i praksa, Beograd: Univerzitet u Beogradu, 2009. 599-610. Štampano.

García Santa-Cecilia, Álvaro. El currículo de español como lengua extranjera (fundamentación metodológica, planificación y aplicación). Madrid: Edelsa, 1995. Impreso.

Gutiérrez Araus, María Luz. Problemas fundamentales de la gramática del español como 2/L. Madrid: Arco/Libros, 2011. Impreso.

Hammer, John H. and Frank A. Rice. A Bibliography of Contrastive Linguistics. Washington: Center for Applied Linguistics, 1965. Print.

Klajn, Ivan. Gramatika srpskog jezika. Beograd: Zavod za udžbenike i nastavna sredstva, 2005. Štampano.

Krstić, Katarina y Tijana Pištignjat. "Análisis de errores en el aprendizaje del español por alumnos que tienen como lengua materna el serbio". Colindancias, 4 (2013): 393-404. Web. 06.06.2013.

Krzeszowski, Tomasz Pawel. Contrastive Languages. The Scope of Contrastive Linguistics. Berlin / New York: Mouton de Gruyter, 1990. Print.

Kurteš, Svetlana. "Contrastive linguistics, a $21^{\text {st }}$ century perspective". Sophia Marmaridou, Kiki Nikiforidou, and Eleni Antonopoulou (eds.). Reviewing Linguistic Thought: Converging Trends for the $21^{\text {st }}$ Century. Berlin: Mouton de Gruyter, 2005. 255-278. Print.

Lado, Robert. Linguistics across Cultures. Ann Arbor: University of Michigan Press, 1957. Print.

Martí Sánchez, Manuel, Inmaculada Penadés Martínez y Ana María Ruiz Martínez. Gramática española por niveles. Madrid: Edinumen, 2008. Impreso.

Martín Morillas, José Manuel. "La Lingüística Contrastiva: desarrollo histórico, abarque, orientaciones, problemas y métodos". Juan de Dios Luque Durán y Antonio Pamies Bertrán (eds.). Panorama de la lingüística actual. Granada: Método Ediciones, 1997. 153-171. Impreso.

Moreno Cabrera, Juan Carlos. Introducción a la Lingüística. Enfoque tipológico y universalista. Madrid: Editorial Síntesis, S.A. 1997. Impreso.

Moreno Cabrera, Juan Carlos. Spanish is Different. Introducción al español como lengua extranjera. Madrid: Editorial Castalia, S.A. 2010. Impreso. 
Pastor Cesteros, Susana. "La concordancia en la interlengua de los aprendices de español como lengua extranjera". Estudios de Lingüística, 15 (2001): 95-125. Web. 15.5.2016.

Penadés Martínez, Inmaculada. "La lingüística contrastiva y el análisis de errores desde la perspectiva del español". Inmaculada Penadés Martínez (coord.). Lingüística contrastiva y análisis de errores (español-portugués y español-chino). Madrid: Editorial Edinumen, 1999. 7-17. Impreso.

Penadés Martínez, Inmaculada. "Las clasificaciones de errores lingüísticos en el marco del análisis de errores". Lingüística en la Red, I (2003): 1-29. Web. 05.11.2003.

Rajić Jelena y Hugo Marcos Blanco. Gramática de la lengua española para serbiohablantes con ejercicios. Beograd: Zavod za udžbenike, 2009. Štampano.

Riđanović, Midhat. „Putevi konstrastivne lingvistike“. Godišnjak Saveza društava za primenjenu lingvistiku Jugoslavije, No. 2 (1978): 81-100. Štampano.

Rubinjoni Strugar, Vlatka. "Análisis de los errores de concordancia en la expresión escrita de los aprendientes serbios de ELE". Lingüística en la RED, No. 11 (2014): 1-34. Web. 18.04.2014.

Santos Gargallo, Isabel. Análisis Contrastivo, Análisis de Errores e Interlengua en el marco de la Lingüística Contrastiva. Madrid: Editorial Síntesis. 1993. Impreso.

Santos Gargallo, Isabel. La enseñanza de segundas lenguas: Análisis de errores en la expresión escrita de estudiantes de español cuya lengua nativa es el serbo-croata. Madrid: Universidad Complutense de Madrid, 1992. Tesis doctoral no publicada.

Stanojčić, Živojin y Ljubomir Popović. Gramatika srpskog jezika za gimnazije i srednje škole. Beograd: Zavod za udžbenike i nastavna sredstva, 2012. Štampano.

Trager, George Leonard. "The Field of Linguistics". Studies in Linguistics: Occasional Papers, Vol. 1 (1949, 3. ${ }^{\text {a }}$ reimp. 1970): 1-8. Print. 


\title{
Vlatka Rubinjoni Strugar
}

\section{THE IMPORTANCE OF COMBINING CONTRASTIVE ANALYSIS AND ERROR ANALYSIS IN CONTRASTIVE SPANISH AND SERBIAN LANGUAGE STUDIES}

\begin{abstract}
Summary
From a theoretical perspective, contrastive analysis results are used for numerous investigations, being especially useful for linguistic typology. From an applied perspective, contrastive linguistics is a practical tool for teaching foreign languages, an area in which it is currently used to develop learning strategies, i.e. to help develop memory and improve understanding. In addition, it fulfils important educational objectives embodied in the elaboration of instruction materials, including contrastive grammars. Error analysis, as a new model of contrastive linguistics, arose from the lack of predictive validity of contrastive analysis. In our work we justify the vitality of contrastive linguistics along with its two methods, contrastive analysis and error analysis, and its suitability for contrastive Spanish and Serbian language studies.
\end{abstract}

Keywords: contrastive linguistics, contrastive analysis, error analysis, learning strategies, Spanish, Serbian. 